\title{
Cost-effectiveness of camrelizumab versus chemotherapy for the treatment of advanced or metastatic esophageal squamous cell carcinoma
}

\author{
Lizong Li, Xuemei Liu, Jing Huang, Yi Liu, Lin Huang, Yufei Feng \\ Department of Pharmacy, Peking University People's Hospital, Beijing, China \\ Contributions: (I) Conception and design: All authors; (II) Administrative support: L Huang, J Huang, Y Liu, Y Feng; (III) Provision of study materials \\ or patients: L Li, X Liu, L Huang, J Huang; (IV) Collection and assembly of data: L Li, X Liu, J Huang, Y Liu, L Huang; (V) Data analysis and \\ interpretation: L Li, X Liu, J Huang, Y Liu, L Huang; (VI) Manuscript writing: All authors; (VII) Final approval of manuscript: All authors. \\ Correspondence to: Yufei Feng. Department of Pharmacy, Peking University People's Hospital, 11 South Xizhimen Street, Beijing 100044, China. \\ Email: fenyufei@126.com.
}

\begin{abstract}
Background: To evaluate the cost-effectiveness of camrelizumab versus chemotherapy for patients with advanced or metastatic esophageal squamous cell carcinoma (ESCC) from the perspective of health system and to provide a basis for health decisions in China.

Methods: A Markov model of 3 health states throughout the lifetime was established based on data from the ESCORT trial. Life-years, quality-adjusted life-years (QALYs), and lifetime costs were estimated. The time horizon of lifetime was 5 years and each model cycle represented 2 months. The cost and utility value adopted a $5 \%$ discount rate per year. One-way sensitivity analysis and probability sensitivity analysis were used to test the robustness of the results.

Results: The results of the cost-effectiveness analysis revealed that the camrelizumab group produced a gain of 2.93 QALY, at a cost of $\$ 37,809.12$ USD, and the chemotherapy group gained 2.85 QALY, at a cost of $\$ 3,7071.52$ USD. Camrelizumab was more cost-effective than chemotherapy for patients with advanced or metastatic ESCC. The results of one-way sensitivity analyses showed that the cost of camrelizumab, cost of chemotherapy and utility of progression-free survival (PFS) state were the top three parameters influencing the model. The probability sensitivity analysis results showed that the results of the basic case analysis were stable.

Conclusions: Under the willingness to pay threshold of three times per capita GDP of China, camrelizumab as second-line treatment could provide more health benefits for advanced or metastatic ESCC in China.
\end{abstract}

Keywords: Cost-effectiveness; Markov; programmed cell death protein 1 (PD-1); esophageal squamous cell carcinoma (ESCC)

Submitted Nov 23, 2021. Accepted for publication Jan 07, 2022.

doi: 10.21037/jgo-21-870

View this article at: https://dx.doi.org/10.21037/jgo-21-870

\section{Introduction}

Esophageal cancer (EC) is a common type of cancer in China and constitutes the sixth leading cause of cancer deaths worldwide (1). It be divided into the histological subtypes of esophageal squamous cell carcinoma (ESCC) and esophageal adenocarcinoma (EAC). In China, more than $90 \%$ are ESCC. For patients with advanced or metastatic ESCC, taxanes, platinum, and 5-fluorouracil $(5 \mathrm{FU})$ are usually used as first-line treatments (2). However, for patients with advanced or metastatic ESCC who have progressed or are intolerant to first-line standard chemotherapy, there are few choices for second-line treatment. In recent years, immunotherapy has presented a new therapeutic strategy for patients with ESCC. The 


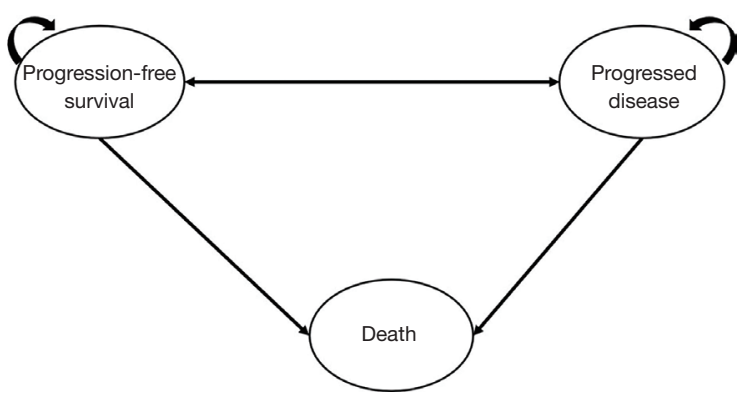

Figure 1 The Markov state transition model.

immune checkpoint programmed cell death protein 1 (PD-1) is expressed in T lymphocytes, B lymphocytes, natural killer cells, and other tumor-infiltrating immune cells (3). By inhibiting the immune checkpoint pathway, the patient's own immune system is used to fight against malignant cells (4). Immune checkpoint inhibitors (ICIs) show good antitumor potential in a variety of human cancers, which can improve the survival rate and quality of life of patients (5). Camrelizumab is a high-affinity, humanized IgG4 monoclonal PD-1 antibody, which can bind to the PD-1 receptor and block its interaction with PD-L1 and PD-L2, and block the immunosuppressive response mediated by PD-1 pathway, including anti-tumor immune response (6). The open-label, phase 3 randomized clinical trial ESCORT reported the efficacy and safety of patients aged $18-75$ years with a histological or cytological diagnosis of advanced or metastatic ESCC treated at 43 hospitals in China (7). The results showed that the median overall survival (OS) of the camrelizumab group was 8.3 [95\% confidence interval (CI): 6.8 to 9.7] months, and the median OS of the chemotherapy group was $6.2(95 \%$ CI: 5.7 to 6.9 ) months [hazard ratio (HR) $0.71,95 \%$ CI: 0.57 to $0.87 ; 2$-sided $\mathrm{P}=0.0010]$. In the evaluation of pharmacoeconomics, compared with other decisionmaking models, the Markov model is especially suitable for simulating the progress of chronic diseases that can be divided into different health states. The present analysis investigated the cost-effectiveness of camrelizumab versus chemotherapy for patients with advanced or metastatic ESCC from the perspective of the Chinese healthcare system by constructing a Markov model. We present the following article in accordance with the CHEERS reporting checklist (available at https://jgo.amegroups. com/article/view/10.21037/jgo-21-870/rc).

\section{Methods}

\section{Model overview}

A Markov model was developed to compare the costeffectiveness of camrelizumab versus chemotherapy for the treatment of advanced or metastatic ESCC. Costeffectiveness analysis (CEA) attempts to determine where more health benefits can be produced at the same cost, or where the same health benefits can be achieved at a lower cost. As shown in Figure 1, the 3 mutually exclusive health states: progression-free disease (PFD), progressive disease (PD), and death constituted the decision-analytic model. All patients started from the state of progression-free survival (PFS) on treatment, then transitioned to PD. Death could occur from any state. The Markov cycle in the model was set at 2 months and the time horizon of the model was 5 years, in view of the short life expectancy of patients with advanced or metastatic ESCC. The primary outputs of the models included the total cost, utility, and incremental cost-effectiveness ratios (ICERs). Costs and outcomes were discounted by $5 \%$ each year, and the willingness-to-pay (WTP) was 3-times of the China's per capita gross domestic product (GDP) in 2020, according to the China Guidelines for pharmacoeconomic evaluations (8). The model was implemented by TreeAge Pro 2021 software (TreeAge, Williamstown, MA, USA), and statistical analyses were performed using R software (version 4.0.5; https://www. r-project.org).

\section{Population and treatment regimens}

The analysis assumed that the target population is advanced or metastatic ESCC and the first-line standard chemotherapy was advanced or intolerant, consistent with the patient characteristics of the ESCORT trial. Treatments were administered as following: (I) camrelizumab group: camrelizumab was intravenously administered on the first day of every 2-week cycle at a dose of $200 \mathrm{mg}$; (II) chemotherapy group: docetaxel was given intravenously over $60 \mathrm{~min}$ on the first day of every 3 weeks at a dose of $75 \mathrm{mg} / \mathrm{m}^{2}$ (7), or irinotecan was infused over $60 \mathrm{~min}$ on the first on the first day of every 2 weeks at a dose of $180 \mathrm{mg} / \mathrm{m}^{2}$. Treatment was continued until disease progression or unacceptable toxicity, patient withdrawal, or by decision of the investigator. The study was conducted in accordance with the Declaration of Helsinki (as revised in 2013). 
Table $1 \mathrm{AIC}$ and BIC for different parameter distributions of OS and PFS

\begin{tabular}{lccccc}
\hline Survival function & Exponential & Weibull & Logistic & Lognormal & Loglogistic \\
\hline AIC for survival distributions of OS in chemotherapy group & $1,761.287$ & $1,736.983$ & $1,713.536$ & $1,721.821$ & $1,713.536$ \\
BIC for survival distributions of OS in chemotherapy group & $1,764.72$ & $1,736.094$ & $1,720.404$ & $1,719.688$ & $1,720.404$ \\
AIC for survival distributions of OS in camrelizumab group & $1,718.234$ & $1,711.386$ & $1,815.845$ & $1,697.217$ & $1,699.7$ \\
BIC for survival distributions of OS in camrelizumab group & $1,721.685$ & $1,718.288$ & $1,822.747$ & $1,704.119$ & $1,706.603$ \\
AIC for survival distributions of PFS in chemotherapy group & $1,225.665$ & $1,142.555$ & $1,179.394$ & $1,116.927$ & $1,110.458$ \\
BIC for survival distributions of PFS in chemotherapy group & $1,229.041$ & $1,149.306$ & $1,186.145$ & $1,123.678$ & $1,117.209$ \\
AIC for survival distributions of PFS in camrelizumab group & $1,565.863$ & $1,566.586$ & $1,736.87$ & $1,507.639$ & $1,502.902$ \\
BIC for survival distributions of PFS in camrelizumab group & $1,569.271$ & $1,573.4$ & $1,743.684$ & $1,514.453$ & $1,509.716$ \\
\hline
\end{tabular}

AIC, Akaike information criterion; BIC, Bayesian information criterion; OS, overall survival; PFS, progression-free survival.

\section{Clinical data inputs}

As individual patient data were not available and followup time was limited, the Getdata Graph Digitizer software (version 2.2.6, https://www.getdata-graph-digitizer.com) was used to extracted survival data, and reconstruction of individual patient data was generated using an algorithm derived by Hoyle et al. (9). Then, these data points were used to fit the following parameter survival functions: Exponential, Weibull, Logistic, Lognormal, and Loglogistic. Combined with visual inspection, the eligible survival function was selected according to the lowest value of the Akaike information criterion (AIC) and the Bayesian information criterion (BIC). The goodness-of-fit results are shown in Table 1. According to the results of AIC and BIC, $\operatorname{loglogistic}$ survival function $S(t)=1 /\left(1+\lambda t^{\gamma}\right)$ was used to fit the Kaplan-Meier (KM) OS and PFS probabilities of the chemotherapy group, and lognormal survival function $\mathrm{S}(\mathrm{t})$ $=1-\Phi\{(\operatorname{lnt}-\mu) / \sigma\}$ and loglogistic survival function were used to fit the KM OS and PFS probabilities of the camrelizumab group, respectively (10). The KM and parameter survival distributions of OS and PFS reconstructed in the model are shown in Figure 2. The transition probability was estimated based on the following formula $\mathrm{tp}(\mathrm{tu})=1-\mathrm{S}(\mathrm{t}) / \mathrm{S}(\mathrm{t}-\mathrm{u})$, where $\mathrm{u}$ was the cycle of the model (11). The key clinical inputs are summarized in Table 2.

\section{Cost and utility inputs}

In this analysis, only direct medical costs, including costs of camrelizumab and chemotherapy, routine follow-ups and monitoring, best supportive care (BSC), and management of treatment-related adverse events (AEs), were considered. All costs were shown in U.S. dollars and adjusted for the 2020 exchange rate ( $\$ 1=¥ 6.8974)$. The costs of camrelizumab and chemotherapy were drawn from China's latest drug bid price (https://www.yaozh.com/). Drug-related costs were calculated based on China's per capita body surface area of $1.72 \mathrm{~m}^{2}(16)$. The costs of routine follow-ups and monitoring, and best supportive care were obtained based on relevant literature (12-15). In calculating the cost of $\mathrm{AE}$ management, we focused only on events of grade 3 or higher. The model assumed that utility is only related to health status, not to therapies. Utility value parameters were derived from published literature and expressed in terms of quality adjustment of life years (QALYs). Table 2 summarizes all model inputs.

\section{Statistical analysis}

In the base-case analysis, CEA was used as the evaluation method to calculate the cost and utility of the chemotherapy group and camrelizumab group respectively. ICERs represents the ratio of the cost difference and the effectiveness difference between different alternatives, and the formula is expressed as:

$$
\operatorname{ICER}=\frac{\Delta \mathrm{C}}{\Delta \mathrm{E}}=\frac{\left(\mathrm{C}_{1}-\mathrm{C}_{2}\right)}{\left(\mathrm{E}_{1}-\mathrm{E}_{2}\right)}
$$

where $\mathrm{C}$ is the cost of treatment and $\mathrm{E}$ is the effectiveness of treatment. To explore the robustness of the costeffectiveness analysis results, one-way sensitivity analyses and probabilistic sensitivity analyses (PSA) were conducted (17). In the one-way sensitivity analyses, assuming that the parameters fluctuate by $20 \%$, the 

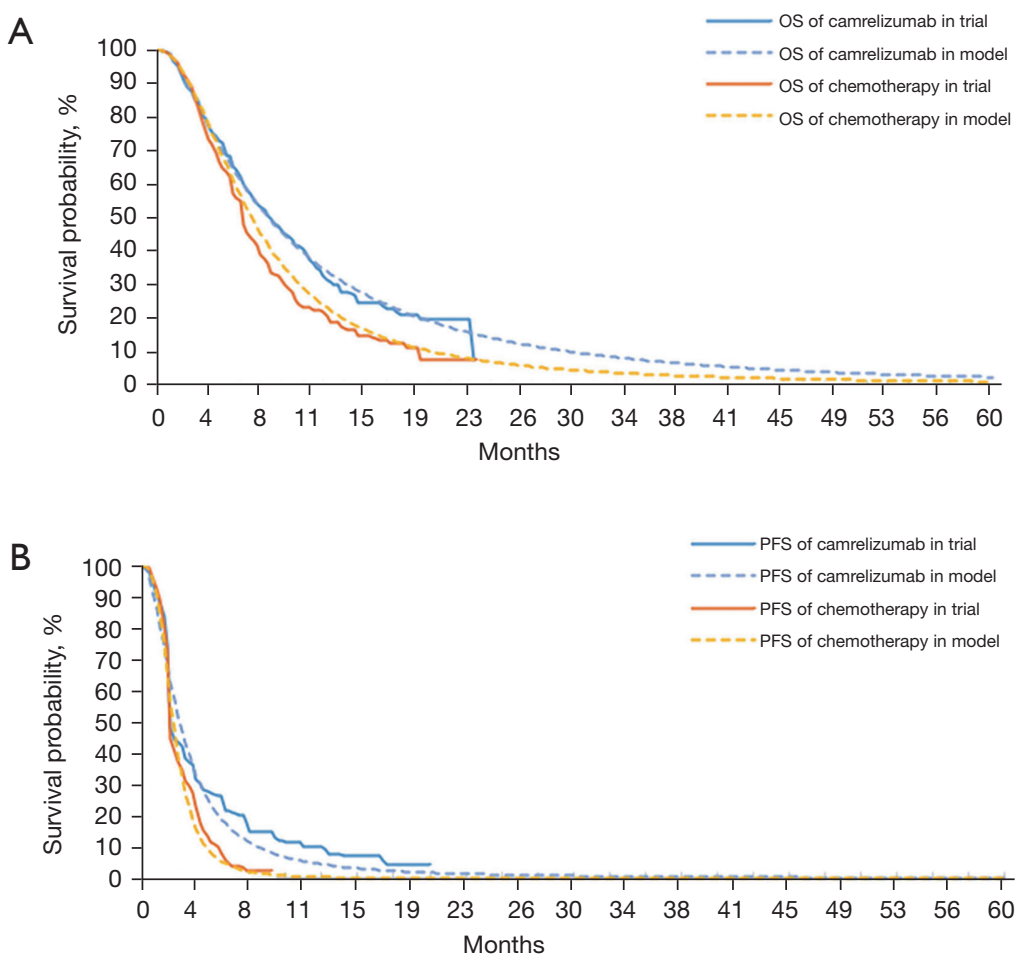

Figure 2 OS curves for the original trial and estimated data (A) and PFS curves for the original trial and model estimated data (B). OS, overall survival; PFS, progression-free survival.

values of the parameters were changed one by one, and the tornado diagram was drawn based on the calculation results (13). To perform the PSA, a second-order Monte Carlo simulation was used to analyze the results through 1,000 random sampling, while the variables varied with a specific distribution pattern (18). Combining parameter constraints and statistical distribution characteristics, we assumed that Gamma distribution was selected for the cost parameters, and the Beta distribution was selected for the utility parameters (19). The PSA was represented by the incremental cost-effectiveness scatter plot and costeffectiveness acceptable curve to verify the uncertainty of the results.

\section{Results}

\section{Base-case analysis}

Within the 5 years of the lifetime analysis, the camrelizumab group gained 2.93 QALY for $\$ 37,809.12$, and the chemotherapy group gained 2.85 QALY for $\$ 37,071.52$. Compared with the chemotherapy group, the ICER of the camrelizumab group was $\$ 9,292.19 /$ QALY. With the
China cost-effectiveness WTP threshold of \$31,510.57/ QALY, camrelizumab had more cost-effectiveness for patients with advanced or metastatic ESCC, compared with chemotherapy. The basic analysis results of the model are given in Table 3.

\section{Sensitivity analysis}

The influence of different parameters on the model results can be shown from the one-way sensitivity analyses. It is suggested in Figure 3 that the cost of camrelizumab and chemotherapy was most sensitive to the model. Other considerable influential parameters in the model were utility of PFS state, probability, and cost of white blood cell count decreased in chemotherapy group, probability and cost of neutrophil count decreased. Changes in other parameters had weak influences on the model results.

The incremental cost-effectiveness scatter plot for camrelizumab versus chemotherapy is shown in Figure 4. A $38.4 \%$ proportion of the scatter points were in the fourth quadrant, indicating that the camrelizumab group had an absolute cost-effectiveness advantage. Concurrently, 56.3\% 
Table 2 Model parameters

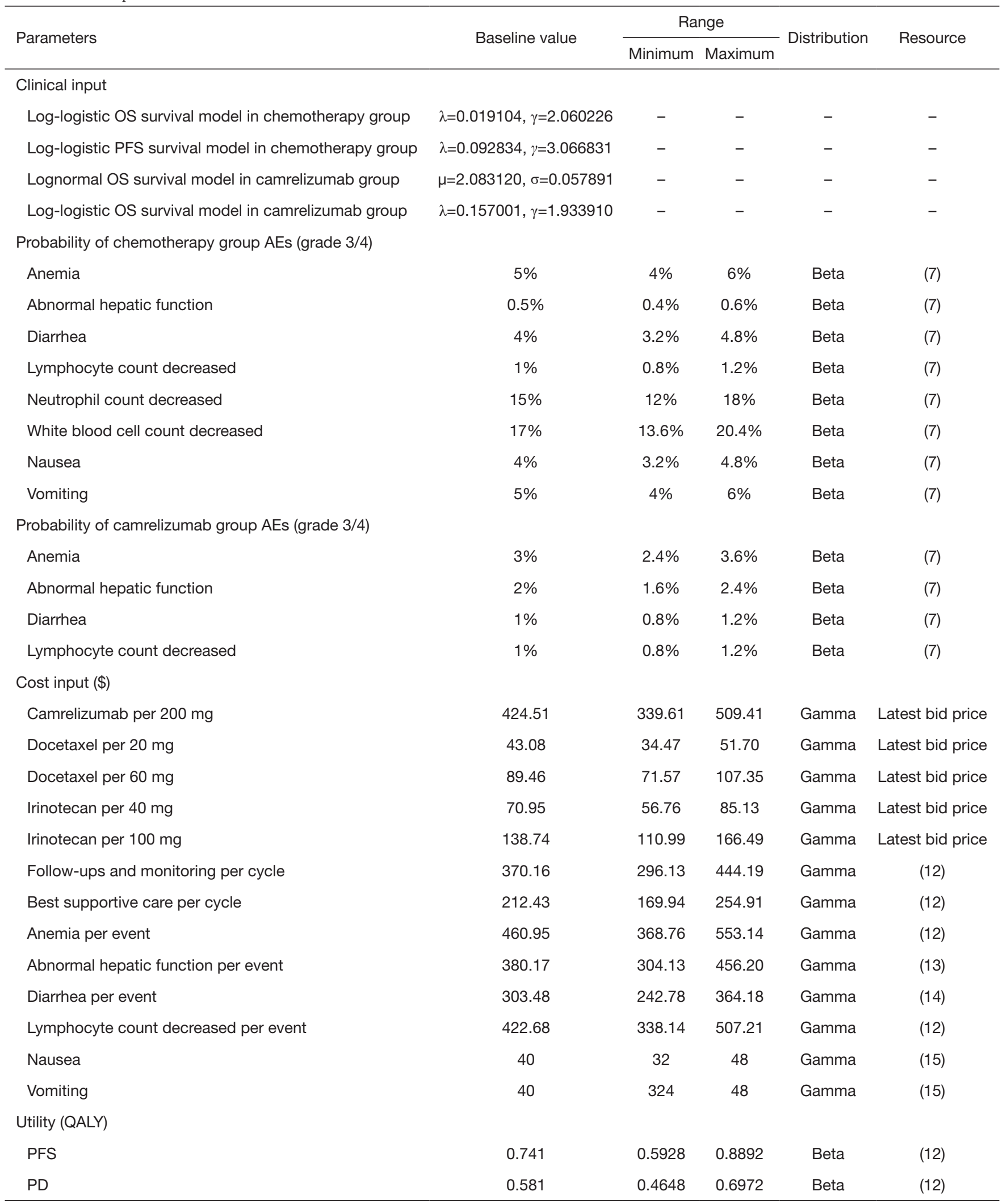

OS, overall survival; PFS, progression-free survival; AEs, adverse events; QALY, quality adjusted life years; PD, progressive disease. 
Table 3 Base-case results of the model

\begin{tabular}{lccccc}
\hline Strategy & Cost, \$ & Incremental cost, \$ & Effectiveness, QALY & Incremental effectiveness, QALY & ICER, \$/QALY \\
\hline Camrelizumab & $37,809.12$ & 737.60 & 2.93 & 0.08 & $9,292.19$ \\
Chemotherapy & $37,071.52$ & - & 2.85 & - & - \\
\hline
\end{tabular}

QALY, quality adjusted life years; ICER, incremental cost-effectiveness ratios.

Tornado diagram-ICER

chemotherapy vs. camrelizumab

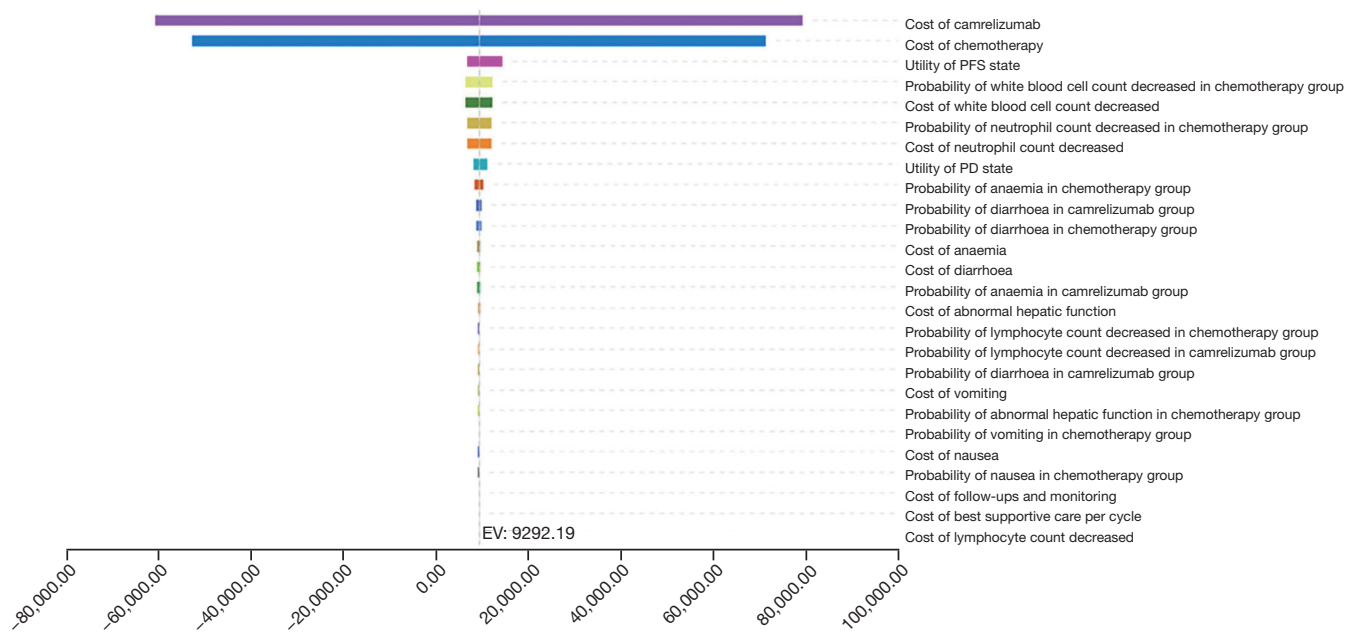

Figure 3 Tornado diagram for one-way sensitivity analyses.

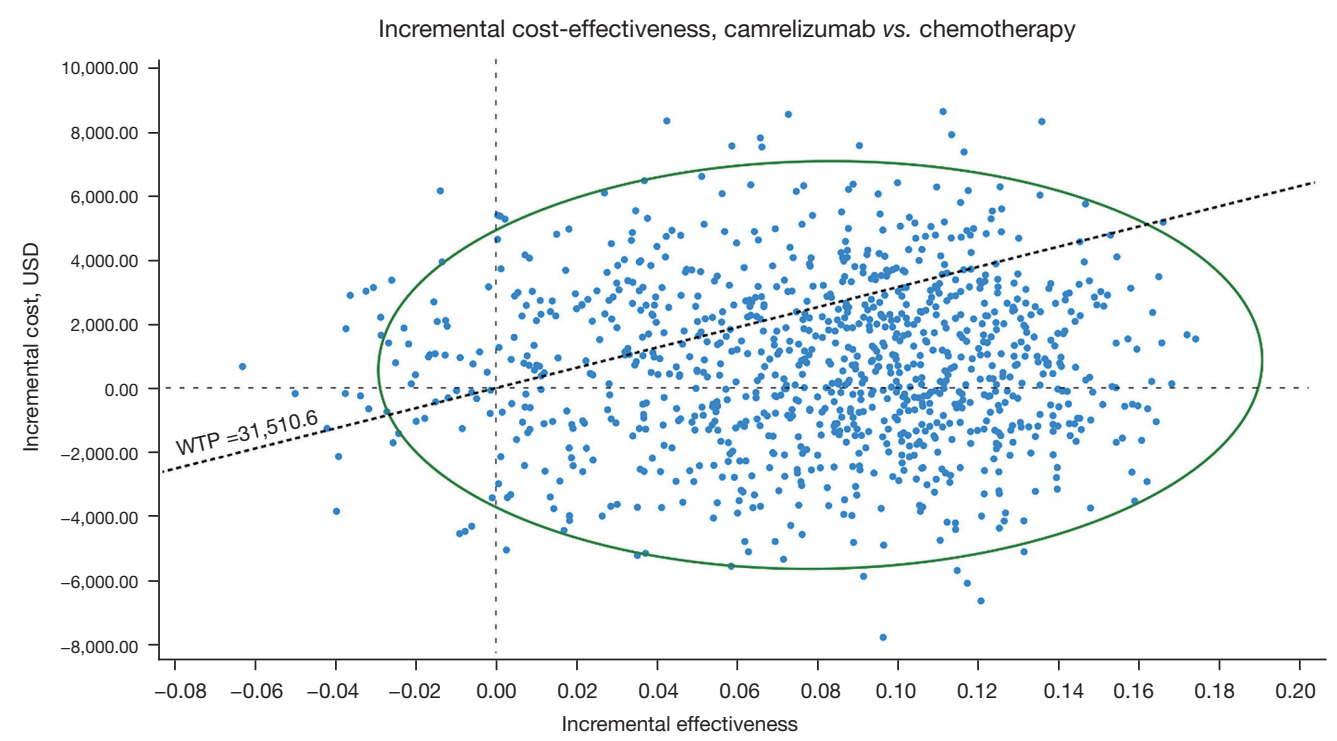

Figure 4 Incremental cost-effectiveness scatter plot diagram for camrelizumab vs. chemotherapy. 


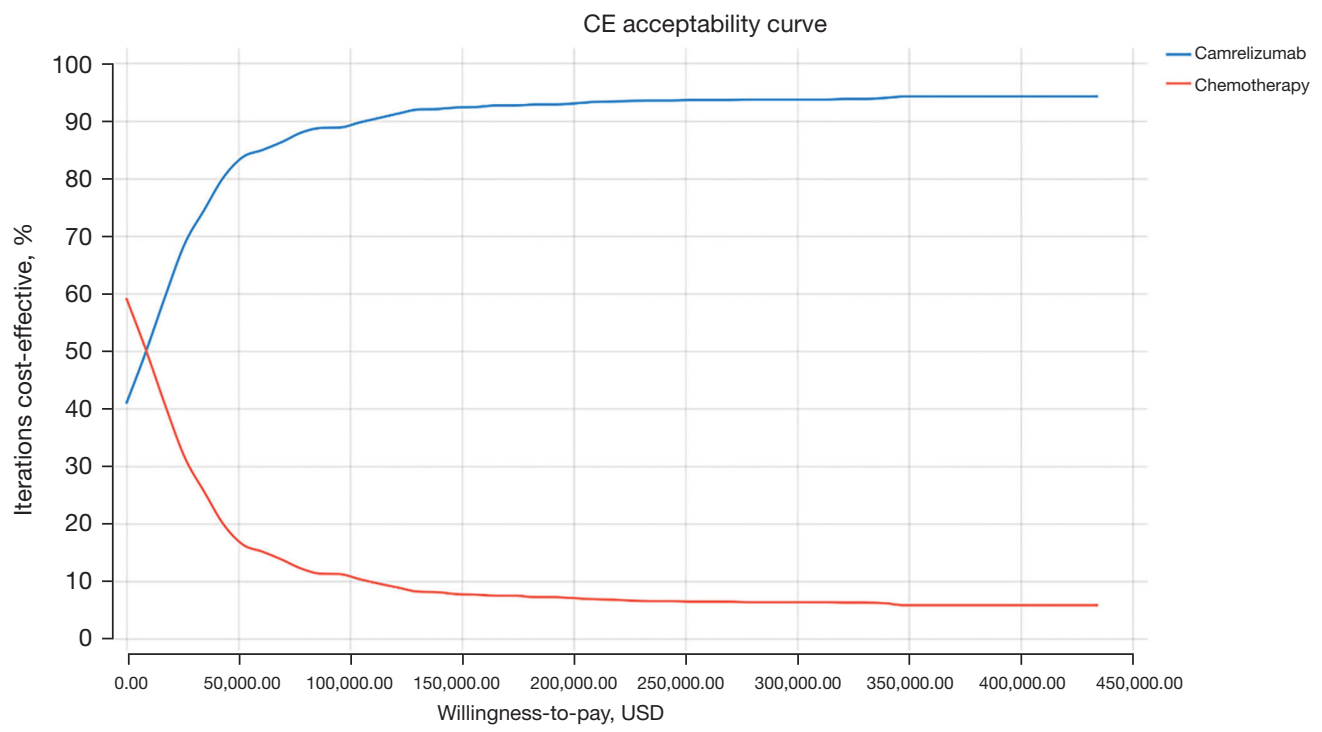

Figure 5 The cost-effectiveness acceptability curves for probabilistic sensitivity analyses. CE, cost-effectiveness.

of the scatter points were in the first quadrant, indicating that patients in the camrelizumab group benefited more, but at the same time the cost was also higher. Generally, more than $50 \%$ of the scatter points were below the WTP threshold, which meant camrelizumab was economically meaningful for patients with advanced or metastatic ESCC.

The Cost-effectiveness acceptability curve is shown in Figure 5. The cost-effectiveness acceptability curve showed a nearly $50 \%$ probability of camrelizumab being a cost-effective strategy at the threshold of $\$ 8,693.64$ per additional QALY gained, and the higher the WTP value, the greater the cost-effectiveness advantage of the camrelizumab group. The results of PSA suggested that the results of the basic case analysis were stable.

\section{Discussion}

China accounts for about half of all ESCC cases on earth due to its large population and high ESCC rates (20). Most patients are diagnosed late and have a poor prognosis. With the continuous breakthrough of anti-tumor efficacy of immunotherapy in various cancers, the treatment of ESCC in recent years has also opened a new era of immunotherapy (21). Camrelizumab has been approved by the China National Medical Products Administration (NMPA) for patients with locally advanced or metastatic ESCC who have previously received first-line chemotherapy and have progressed or become intolerable. It has become
China's first immunotherapy drug for esophageal cancer in the whole population. In developing countries, the high price of anti-cancer drugs may put cancer patients facing serious economic risks. As immunotherapy plays an increasingly important role in cancer treatment, clinicians should consider both the efficacy and the high cost when making treatment decisions. Although there have been previous pharmacoeconomic evaluations of camrelizumab, based on the previous price, camrelizumab was deemed not economical for patients with ESCC (22). However, camrelizumab entered the National Reimbursement Drug List (NRDL) in 2020. After the efforts of all parties, the price reduction will eventually reach $85 \%$. In this study, we updated part of the model input data, re-evaluated the cost-effectiveness of camrelizumab, and drew different research conclusions. Based on the newly announced winning bid price, combined with the published literature on advanced or metastatic ESCC and the current status of Chinese medical resources, we conducted a costeffectiveness analysis of camrelizumab, aiming to provide a reference for clinical rational use of drugs and related health decisions. Although the treatment cost of camrelizumab after the sharp price reduction is still higher than that of the chemotherapy group, it has a certain cost-effectiveness advantage under the threshold of 3 times China's per GDP. The price adjustment of camrelizumab effectively reduced the economic burden of the healthcare system and provided more value to patients. The epidemiology of esophageal 
cancer in China has significant regional differences, and the main high-incidence areas are located in rural and mountainous areas (23), where the majority of people have lower incomes. According to the Statistical Communiqué of the People's Republic of China on the 2020 National Economic and Social Development, the per capita disposable income of rural residents in China is $\$ 2,483.69$ (24). Compared with the chemotherapy group, the ICER value of camrelizumab was $\$ 9,292.19 /$ QALY. As far as rural residents were concerned, the burden of disease was still relatively heavy. How to meet the most basic needs of the population with limited medical insurance resources, deepen the reform of medical service prices, and increase the availability of high-priced drugs remain key public health issues to be considered in decision-making. It should be mentioned that this study also had several limitations. First, the model established in this study was based on specific clinical trials and an ideal state for ESCC development. Due to the limitation of data sources, this study only established a 3 -state Markov model, which can be added to the state of the model and make the model closer to the complex ESCC progression. Second, the utility value parameter came from the European population in the previously published literature, and there may be a deviation for the Chinese population. The results of this study revealed that the priceadjusted camrelizumab has certain pharmacoeconomic value in the treatment of advanced or metastatic ESCC. It is hoped that more real-world studies based on camrelizumab and health outcome evaluation scales for the Chinese population can be carried out in the future, so as to provide more references for medical decision-making departments.

\section{Acknowledgments}

Funding: This study was supported by Bethune Charitable Foundation of Pharmaceutical Research Capacity Building Project (No. B-19-H-20200622).

\section{Footnote}

Reporting Checklist: The authors have completed the CHEERS reporting checklist. Available at https://jgo. amegroups.com/article/view/10.21037/jgo-21-870/rc

Data Sharing Statement: Available at https://jgo.amegroups. com/article/view/10.21037/jgo-21-870/dss

Conflicts of Interest: All authors have completed the ICMJE uniform disclosure form (available at https://jgo.amegroups. com/article/view/10.21037/jgo-21-870/coif). The authors have no conflicts of interest to declare.

Ethical Statement: The authors are accountable for all aspects of the work in ensuring that questions related to the accuracy or integrity of any part of the work are appropriately investigated and resolved. The study was conducted in accordance with the Declaration of Helsinki (as revised in 2013).

Open Access Statement: This is an Open Access article distributed in accordance with the Creative Commons Attribution-NonCommercial-NoDerivs 4.0 International License (CC BY-NC-ND 4.0), which permits the noncommercial replication and distribution of the article with the strict proviso that no changes or edits are made and the original work is properly cited (including links to both the formal publication through the relevant DOI and the license). See: https://creativecommons.org/licenses/by-nc-nd/4.0/.

\section{References}

1. Yang $\mathrm{YM}$, Hong $\mathrm{P}, \mathrm{Xu} \mathrm{WW}$, et al. Advances in targeted therapy for esophageal cancer. Signal Transduct Target Ther 2020;5:229.

2. Harada K, Rogers JE, Iwatsuki M, et al. Recent advances in treating oesophageal cancer. F1000Res 2020;9:eF1000 Faculty Rev-1189.

3. Rong L, Liu Y, Hui Z, et al. PD-L1 expression and its clinicopathological correlation in advanced esophageal squamous cell carcinoma in a Chinese population. Diagn Pathol 2019;14:6.

4. Joyce JA, Fearon DT. T cell exclusion, immune privilege, and the tumor microenvironment. Science 2015;348:74-80.

5. Ai L, Chen J, Yan H, et al. Research Status and Outlook of PD-1/PD-L1 Inhibitors for Cancer Therapy. Drug Des Devel Ther 2020;14:3625-49.

6. Markham A, Keam SJ. Camrelizumab: First Global Approval. Drugs 2019;79:1355-61.

7. Huang J, Xu J, Chen Y, et al. Camrelizumab versus investigator's choice of chemotherapy as second-line therapy for advanced or metastatic oesophageal squamous cell carcinoma (ESCORT): a multicentre, randomised, open-label, phase 3 study. Lancet Oncol 2020;21:832-842.

8. Liu GE, Hu SL, Wu JH, et al. China guidelines for pharmacoeconomic evaluations 2020 (Chinese-English Version). Beijing, China: China Market Press, 2020. 
9. Hoyle $M W$, Henley $W$. Improved curve fits to summary survival data: application to economic evaluation of health technologies. BMC Med Res Methodol 2011;11:139.

10. Djalalov S, Beca J, Ewara EM, et al. A Comparison of Different Analysis Methods for Reconstructed Survival Data to Inform Cost-Effectiveness Analysis. Pharmacoeconomics 2019;37:1525-36.

11. Diaby V, Adunlin G, Montero AJ. Survival modeling for the estimation of transition probabilities in model-based economic evaluations in the absence of individual patient data: a tutorial. Pharmacoeconomics 2014;32:101-8.

12. Zhang PF, Xie D, Li Q. Cost-effectiveness analysis of nivolumab in the second-line treatment for advanced esophageal squamous cell carcinoma. Future Oncology 2020;16:1189-98.

13. Shi F, Meng R, Wang Z, et al. Cost-effectiveness analysis of applying camrelizumab as second-line therapy for the treatment of advance hepatocellular carcinoma. Chinese Health Economics 2021;40:62-5.

14. Zhang Y, Zeng X, Wan X, et al. Cost utility analysis of atezolizumab in the treatment of metastatic non-small cell lung cancer. Chinese Journal of Hospital Pharmacy 2021;41:828-32.

15. Li S, Peng L, Tan C, et al. Cost-Effectiveness of ramucirumab plus paclitaxel as a second-line therapy for advanced gastric or gastro-oesophageal cancer in China. PloS One 2020;15:e0232240.

16. Wu B, Dong B, Xu Y, et al. Economic evaluation of firstline treatments for metastatic renal cell carcinoma: a costeffectiveness analysis in a health resource-limited setting. PLoS One 2012;7:e32530.

Cite this article as: Li L, Liu X, Huang J, Liu Y, Huang L, Feng Y. Cost-effectiveness of camrelizumab versus chemotherapy for the treatment of advanced or metastatic esophageal squamous cell carcinoma. J Gastrointest Oncol 2022;13(1):40-48. doi: 10.21037/jgo-21-870
17. Andronis L, Barton P, Bryan S. Sensitivity analysis in economic evaluation: an audit of NICE current practice and a review of its use and value in decision-making. Health Technol Assess 2009;13:iii, ix-xi, 1-61.

18. Claxton K. Exploring uncertainty in cost-effectiveness analysis. Pharmacoeconomics 2008;26:781-98.

19. Su D, Wu B, Shi L. Cost-effectiveness of Atezolizumab Plus Bevacizumab vs Sorafenib as First-Line Treatment of Unresectable Hepatocellular Carcinoma. JAMA Netw Open 2021;4:e210037.

20. Abnet CC, Arnold M, Wei WQ. Epidemiology of Esophageal Squamous Cell Carcinoma. Gastroenterology 2018;154:360-73.

21. Fatehi Hassanabad A, Chehade R, Breadner D, et al. Esophageal carcinoma: Towards targeted therapies. Cell Oncol (Dordr) 2020;43:195-209.

22. Yang F, Fu Y, Kumar A, et al. Cost-effectiveness analysis of camrelizumab in the second-line treatment for advanced or metastatic esophageal squamous cell carcinoma in China. Ann Transl Med 2021;9:1226.

23. Liang H, Fan JH, Qiao YL. Epidemiology, etiology, and prevention of esophageal squamous cell carcinoma in China. Cancer Biol Med 2017;14:33-41.

24. National Bureau of Statistics of China (Internet). Statistical Communiqué of the People's Republic of China on the 2020 National Economic and Social Development; 2021 (cited 2021 Jul 12). Available online: http://www.stats.gov.cn/english/PressRelease/202102/ t20210228_1814177.html

(English Language Editor: J. Jones) 